\title{
Erosion autour de structures côtières
}

\author{
Larroudé ${ }^{1} \mathrm{Ph}$. et Mory ${ }^{2} \mathrm{M}$.
}

1. Maitre de Conférences

Laboratoire des Ecoulements Géophysiques et Industriels, BP53,

38041 Grenoble Cédex 9, tel: 334768250 60; fax 334768250 01;

email:Larroude@hmg.inpg.fr

2. Professeur

ENSGTI, Université de Pau et des Pays de l'Adour, me Jules Ferry,

64000 Pau, tel: 335597221 32; fax 33559722081

email:mathieu.mory@univ-pau.fr

\section{Résumé}

Dans cet article, nous allons mettre l'accent sur les processus d'érosion locale et globale à partir de deux séries d'études expérimentales : (1) les processus d'érosion autour d'un groupe de piles, (2) les processus d'érosion autour d'un cylindre vertical dans la zone de déferlement. La présente étude a été menée au sein du programme européen SCARCOST (EC-MAST3). L'érosion formée autour des groupes de piles a été caractérisée pour différentes conditions de houle, différents diamètres de pile et différents espacements entre les piles, et pour différents arrangements de piles. Les expériences ont été faites par le LEGI dans un bassin à vagues de $9 \mathrm{~m} \times 30 \mathrm{~m}$ au Laboratoire d'Hydraulique de France (LHF) à Grenoble. L'étude a permis de mettre en évidence que l'érosion globale apparaît en même temps que le processus local dans les différentes conditions expérimentales. Des rides de fonds sont observées dans la plupart des tests.

\begin{abstract}
The paper will focus on a discussion of local and global scour processes, by considering two test cases : (1). scour processes around in a group of piles, (2). scour processes around a vertical cylinder located in the surf zone. The present study was conducted in the framework of the project SCARCOST, partially funded by the EC-MAST 3 program. The scour produced by waves around piles forming groups has been characterised for different wave conditions, different pile diameters and gaps between piles, different pile arrangements and different orientations of wave propagation to the pile groups. The experiments were conducted in a $9 \mathrm{~m}$ by $30 \mathrm{~m}$ wave basin at Laboratoire d'Hydraulique de France (LHF) in Grenoble and operated by LEGI. Global scour overcome local scour processes in a group of piles. Wave ripples were observed for most conditions all over the bed. The respective scaling of wave ripple depth and scour depth are discussed. The occurrence of deep global scour around a single pile located in the surf zone will be considered.
\end{abstract}




\section{Introduction}

Depuis plusieurs années, suite aux travaux de Larras (1972) et d'Herbisch (1991), l'érosion autour de structures verticales cylindriques a été étudiée par différents auteurs, comme le rappelle dans son livre R. Whitehouse (1998). Le nombre de Keulegan-Carpenter $K C=U_{m} T / D$, où $U_{m}, T$ et $D$ désignent respectivement l'amplitude de la vitesse d'oscillation près du fond, la période de la houle et le diamètre des piles, est le paramètre principal gouvernant les processus d'érosion autour des structures côtières. Le trou formé par l'érosion autour d'une pile n'apparait que lorsque $\mathrm{KC}$ est suffisamment grand (quand le diamètre de la pile est petit devant la longueur $d$ 'onde de la houle). Les principales caractéristiques de l'érosion locale sont présentées sur la Figure 1. La profondeur S est l'écart entre le pied de la pile au plus profond du trou et le niveau du fond loin de la pile.

La propagation de la houle n'est pas affectée par la présence des piles sauf dans le proche voisinage de celles-ci où Sumer et al. (1992) ont identifié le lâcher de tourbillons alternés d'axes parallèles à celui du cylindre et la présence de tourbillons en fer à cheval (horseshoe vortex) comme responsables de l'apparition d'une profonde érosion locale. Ils ont ainsi mis en évidence une relation entre la profondeur de l'érosion locale et le nombre de Keulegan-Carpenter.

Dans cet article, nous allons mettre l'accent sur les processus d'érosion locale et globale à partir de deux séries d'études expérimentales : (1) les processus d'érosion autour d'une pile isolée et au sein d'un groupe de piles, (2) les processus d'érosion autour d'un cylindre verticale dans la zone de déferlement. La présente étude a été menée au sein du projet européen SCARCOST (EC-MAST3). L'érosion formée autour des groupes de piles a été caractérisée pour différentes conditions de houle, différents diamètres et espacements entre les piles, pour différents arrangements de piles et pour différentes directions de propagation de la houle.

\section{Méthode expérimentale}

Les expériences ont été réalisées par le LEGI dans un bassin à vagues de $9 \mathrm{~m} \mathrm{x}$ $30 \mathrm{~m}$ du Laboratoire d'Hydraulique de France (LHF). Les conditions de houle régulière fournissent dans nos expériences une gamme de valeurs de $\mathrm{KC}$ allant de 1 à 30 . Les groupes de pile étaient initialement sur un fond sableux (taille des grains : $D_{50}=320 \mu \mathrm{m}$ ) formant une plage de pente $1 / 40$.

Trois différents type de piles ont été utilisés. Pour les expériences avec les groupes de piles, nous avons étudié les deux diamètres $D=3 \mathrm{~cm}$ et $\mathrm{D}=6.3 \mathrm{~cm}$ et pour les cas avec la pile isolée un diamètre $\mathrm{D}=40 \mathrm{~cm}$. Dans les séries avec les groupes de piles, un autre paramètre important, est l'écart $\mathrm{G}$ séparant deux piles dans chaque direction. Nous avons considéré les deux rapports $G / D=1$ et $G / D=2$. Nous avons également fait varier l'angle d'incidence de la houle par rapport à 
l'orientation du groupe de piles en faisant tourner l'ensemble de l'angle voulu par rapport au batteur. Les angles $\alpha$ étudiés sont $\alpha=0^{\circ}, 45^{\circ}$ et $90^{\circ}$.

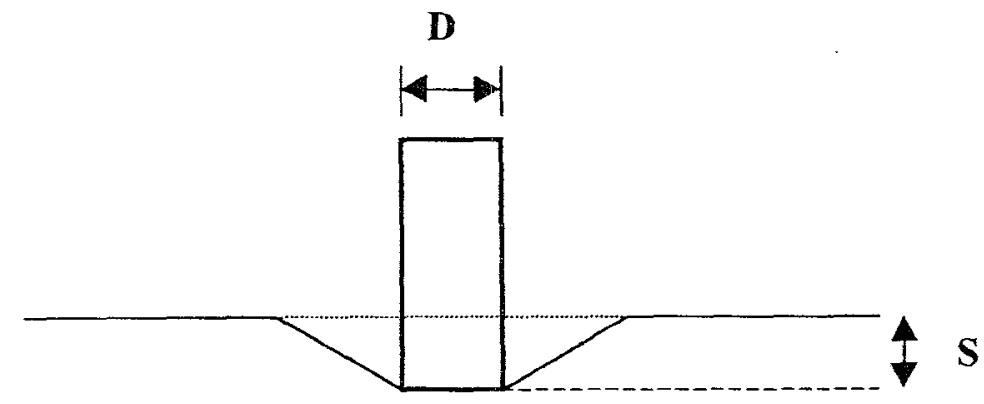

Figure 1. Définition de la profondeur d'érosion, Scour depth definition for local scour.

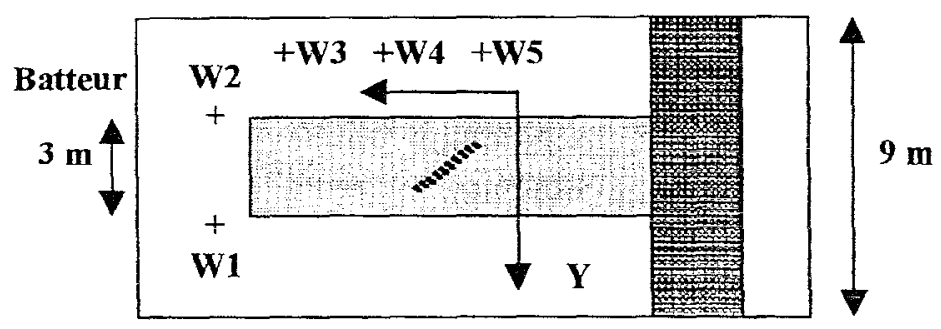

Figure 2. Dispositif expérimental, Layout of the experimental set-up

Les conditions de houle sont les mêmes pour toutes les expériences avec un groupe de piles. La houle produite par le batteur est unidirectionnelle régulière avec une période $\mathrm{T}=1.5 \mathrm{~s}$ et une hauteur $\mathrm{H}_{\mathrm{m}, \mathrm{d}}=0.11 \mathrm{~m}$ dans une profondeur d'eau au repos de $0.4 \mathrm{~m}\left(\mathrm{H}_{\mathrm{m}, \mathrm{d}}\right.$ est la hauteur des vagues à la profondeur moyenne $\mathrm{h}$ de la couche d'eau dans le groupe de piles). Ces conditions de houle ont été choisies pour que, malgré le changement des propriétés des vagues lorsqu'elles se propagent vers la plage, elles engendrent à proximité des piles un écoulement avec un nombre de Keulegan-Carpenter suffisamment grand. Le déferlement de cette houle se produit à une distance de environ une longueur d'onde à l'arrière du groupe de piles en direction du rivage.

Les mesures des hauteurs de vagues ont été réalisées grâce à des jauges localisées à différents endroits à l'intérieur du bassin. Leurs positions (w1 à w5) sont indiquées sur la figure 2 où les zones grisées représentent le sable sur une largeur de $3 \mathrm{~m}$ et sur une longueur de $16 \mathrm{~m}$ (le début de la zone de sable se situant à $4 \mathrm{~m}$ du batteur.

Les deux jauges w1 et w2, positionnées à une distance de $1.7 \mathrm{~m}$ du générateur de vagues, servent à vérifier que les vagues sont cylindriques à l'origine. Les jauges w3, w4, w5 sont alignées parallèlement à la direction de propagation des vagues. Elles sont utilisées pour caractériser l'évolution des vagues sur la plage (voir Mory et al. (1999)). Le nombre de Keulegan-Carpenter, issu de ces 
expériences $(\mathrm{KC}=\mathrm{Um}$. T/D) est proche de la valeur calculée à partir de la théorie linéaire : $\mathrm{KC}=\pi \mathrm{H}_{\mathrm{m}, \mathrm{d}} / \mathrm{Dsh}(\mathrm{kh})$ où $\mathrm{k}$ est le nombre d'onde.

Les quantités $\mathrm{k}$ et $\mathrm{H}_{\mathrm{m} \text {, d }}$ sont issues de la théorie linéaire de la réfraction, pour une hauteur initiale des vagues de $\mathrm{H}_{\mathrm{m}, \mathrm{d}}=0.11 \mathrm{~m}$ dans une profondeur d'eau de $0.4 \mathrm{~m}$.

Pour une pile de diamètre $\mathrm{D}=6.3 \mathrm{~cm}$, la valeur de $\mathrm{KC}$ évaluée par la théorie précédente est de 7.2 tandis que les mesures donnent $\mathrm{KC}=7.9$. Pour un diamètre de $3 \mathrm{~cm}$, on obtient respectivement des valeurs théoriques et expérimentales de 15.2 et 16.5. La théorie linéaire évalue de façon satisfaisante le nombre de Keulegan-Carpenter. Les écarts raisonnables constatés peuvent être dû soit à la faible non-linéarité des houles constatées sur les enregistrements, soit aux modifications du profil de plage produit au cours d'une expérience.

La durée moyenne de nos expériences, de l'ordre de 9 heures, correspond à environ 22000 périodes de vagues. A l'issue de chaque expérience, les transformations du fond ont été mesurées à l'aide d'une sonde acoustique qui relève la cote du fond sur un domaine de $3 \mathrm{~m}$ par $3 \mathrm{~m}$ avec un pas de $1 \mathrm{~cm}$ dans chaque direction du plan de coordonnées. Pour toutes les représentations de cet article, le sens de propagation des vagues est suivant les $\mathrm{x}$ négatifs. L'ensemble des expériences effectuées est résumé dans le tableau suivant:

\begin{tabular}{|c|c|c|c|c|}
\hline & $\mathrm{D}$ & $\mathrm{KC}$ & $\alpha$ & $\mathrm{G} / \mathrm{D}$ \\
\hline Groupe de Piles & $3 \mathrm{~cm}$ & 7.9 & $0^{\circ}, 45^{\circ}, 90^{\circ}$ & 1 et 2 \\
\hline Groupe de Piles & $6.3 \mathrm{~cm}$ & 16.5 & $0^{\circ}, 45^{\circ}, 90^{\circ}$ & 1 et 2 \\
\hline Pile isolée & $40 \mathrm{~cm}$ & 1.2 & $0^{\circ}$ & - \\
\hline
\end{tabular}

\section{Résultats}

\subsection{Généralités}

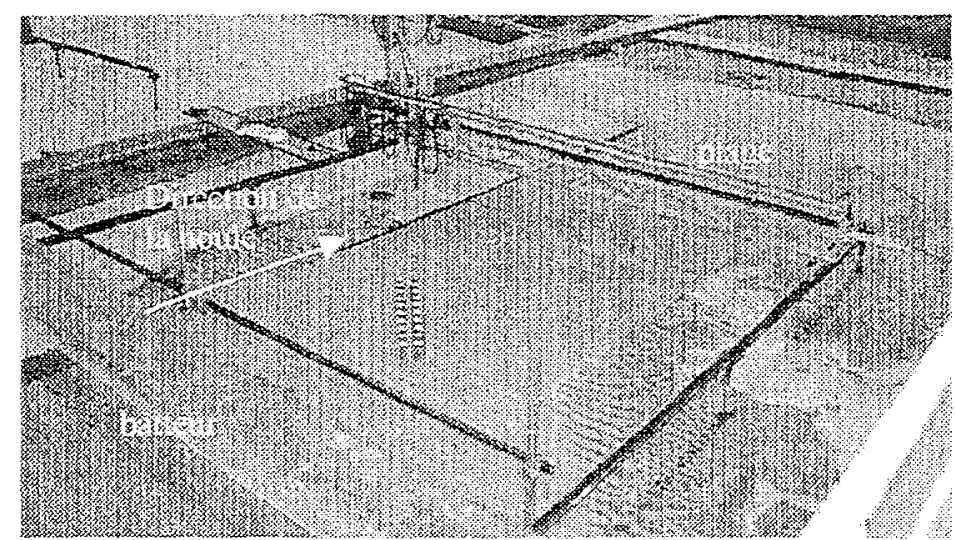

Figure 3. Vue générale du bassin, General view of the wave tank

La figure 3 montre une vue générale du bassin prise à la fin d'une expérience. Cette image montre que le transport du sable durant les expériences se 
fait en direction du rivage, au voisinage duquel on observe une accumulation de sable. On remarque également un dépôt de sable sur les côtés latéraux du bassin qui indique le positionnement des courants de retour.

La Figure 4 montre deux photographies montrant la forme des fonds au voisinage d'un groupe de piles, prises à l'issue de deux expériences pour lesquelles seul le diamètre des piles diffère. Ces images nous montrent une érosion locale et globale pour deux valeurs de KC. L'érosion locale n'est pas le phénomène principal des changements de fond, même si nous pouvons observer que les trous autour de certaines piles sont plus profonds pour $K C=16,5(D=3 \mathrm{~cm})$ que pour $\mathrm{KC}=7,9(\mathrm{D}=6,3 \mathrm{~cm})$. Cette tendance est en accord, qualitativement et en ordre de grandeur, avec les résultats de Sumer et al. (1992) sur la profondeur des trous d'érosion autour d'une pile isolée.

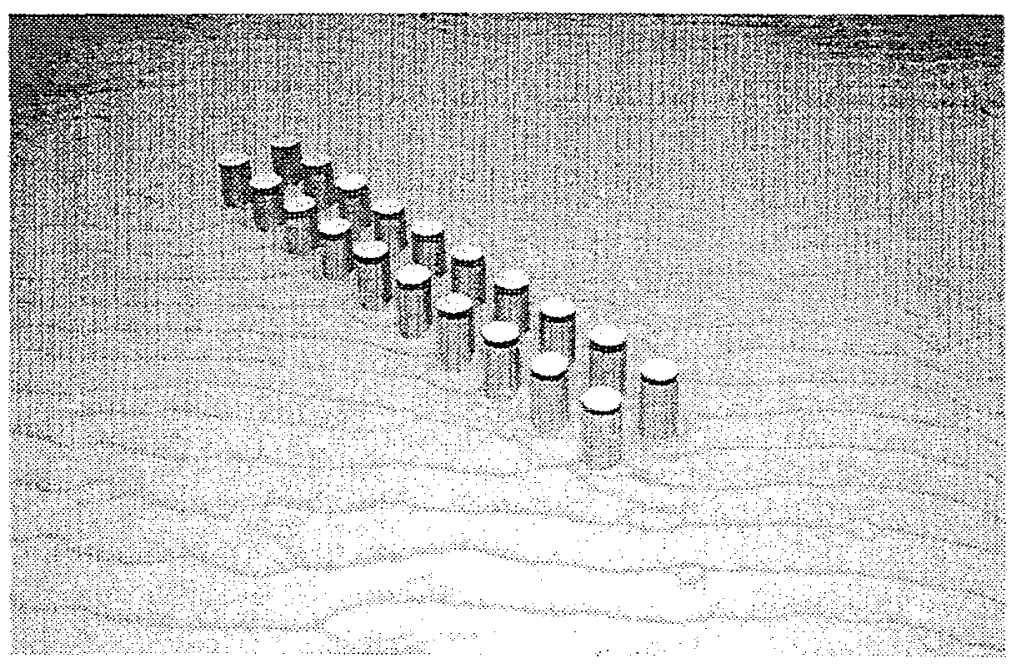

$4 a \cdot D=6.3 \mathrm{~cm}, \mathrm{G} / \mathrm{D}=1, \alpha=45^{\circ}, \mathrm{KC}=7.9$

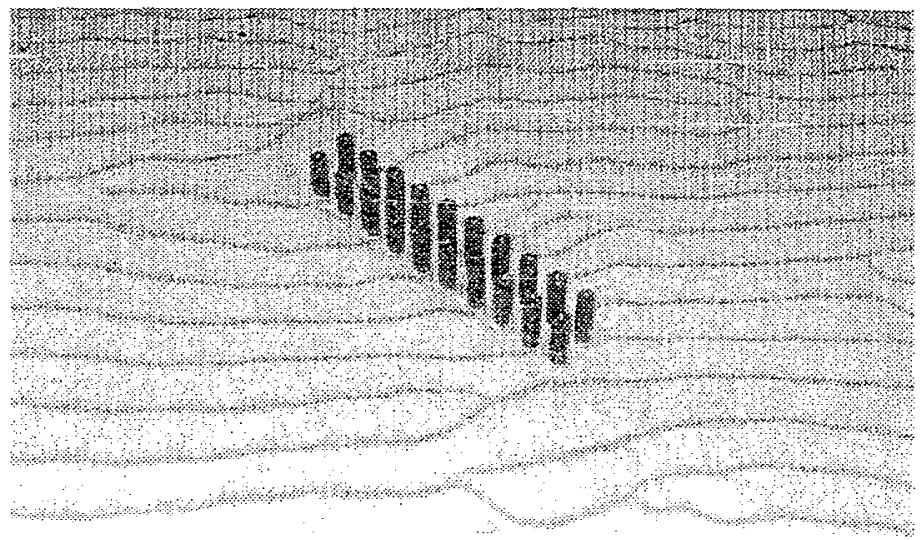

4b. $\mathrm{D}=3 \mathrm{~cm}, \mathrm{G} / \mathrm{D}=1, \alpha=45^{\circ}, \mathrm{KC}=16.5$

Figure 4. Forme des fonds au voisinage d'un groupe de piles, Bed level changes inside and in the vicinity of the piles group 
Des rides sur le fond ont été observées dans toutes nos expériences, dont la profondeur moyenne était de l'ordre de grandeur de la profondeur des trous d'érosion. On peut faire remarquer, avec Sutherland et Whitehouse (1998), que l'échelle des rides est indépendante du diamètre des piles. Sumer et al. (1992) l'avaient constaté par les nombreuses expériences qu'ils avaient réalisées, pour lesquelles la longueur des rides variaient entre un demi diamètre à plusieurs diamètres de pile. Leur conclusion étaient que les rides sont un phénomène indépendant du processus d'érosion. La photographie de la Figure 4 montre que le chant de rides n'est que peu affecté par le groupe de piles à $45^{\circ}$. En particulier, on remarque que c'est seulement entre les piles que les modifications sont significatives. Le principal effet du groupe de pile est de produire des modifications morphodynamiques de grande échelle autour de lui. Dans le cas des petits diamètres, le groupe de piles se trouve au sein d'un seul grand trou alors que pour les piles plus grosses le niveau moyen du fond augmente entre les piles. Ceci montre qu'une érosion locale et une érosion globale sont simultanément produites dans nos expériences.

\subsection{Modifications du fond sableux}

La bathymétrie du fond sableux a été mesurée autour des groupes de piles et au sein de ces groupes à la fin de chacune des expériences. Nous présentons ici seulement quelques résultats significatifs pour $G / D=1$. Des conclusions similaires sont obtenues pour $\mathrm{G} / \mathrm{D}=2$. Nous comparons les résultats pour les deux valeurs de $\mathrm{KC}=7,9$ et $\mathrm{KC}=16,5$ pour différentes orientations du groupe de piles par rapport à la direction de propagation des vagues. Les différentes cartes d'iso-valeurs de bathymétrie nous permettent d'obtenir différentes informations pour plusieurs échelles spatiales. Les changements morphologiques de grandes échelles sont dus principalement à deux causes. La première, et la plus naturelle, est la modification $\mathrm{du}$ profil de la plage résultant notamment du déferlement des vagues indépendamment de la présence des piles. La deuxième cause de changement à grande échelle est due à la diffraction de vagues au passage du groupe de piles. Ces modifications se rajoutent aux premières

Les plus petites échelles concernent les rides, qui recouvrent l'ensemble du lit, et l'érosion locale autour des piles. Pour analyser correctement ces différentes échelles, nous avons appliqué une procédure de filtration spatiale aux champs de mesures bathymétriques. Les différentes étapes de ce filtrage sont décrites sur la Figure 5. La Figure 5a montre la bathymétrie directement issue des données en fin d'expérience. Les niveaux des contours représentent la différence avec l'état initial et leurs valeurs négatives montrent les zones érodées.

Un filtre passe-bas est appliqué sur ces données pour obtenir les variations de la bathymétrie à grande échelle, appelée bathymétrie morphodynamique. Pour le cas de la Figure $5 b$, l'angle d'incidence des vagues par rapport au groupe de piles est de $45^{\circ}$ et la diffraction des vagues modifie de façon non négligeable la morphodynamique grande échelle. On observe également un dépôt de sable au sein et autour des piles, d'environ 20 à $30 \mathrm{~mm}$ d'épaisseur. 


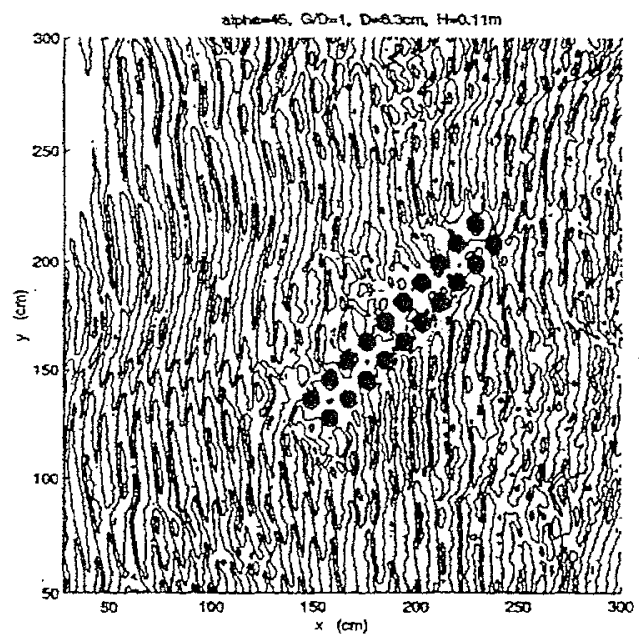

a)

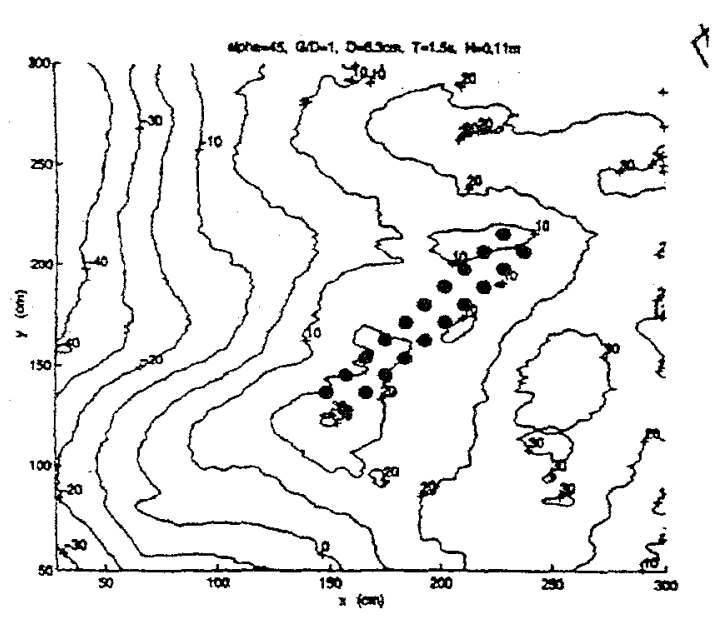

b)

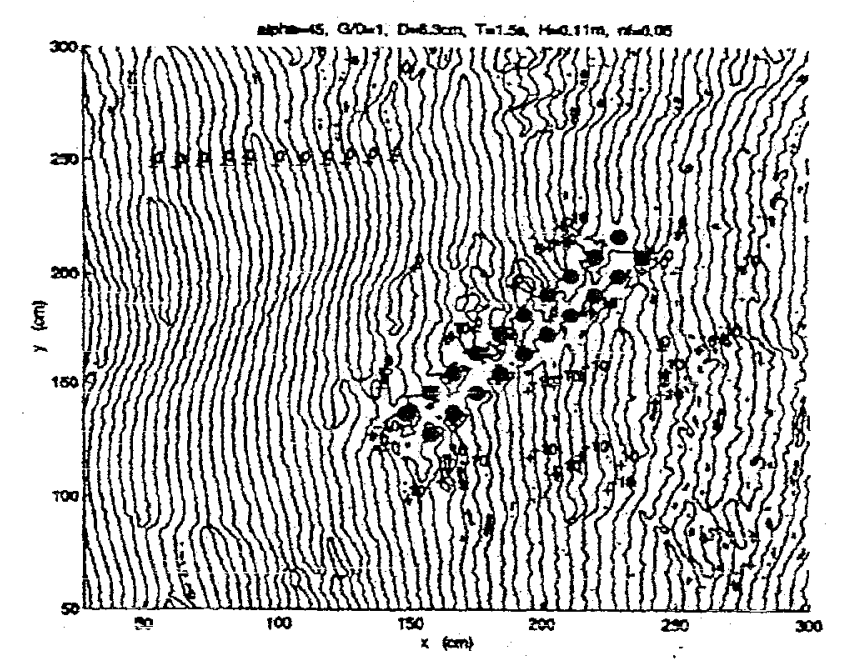

c).

Figure 5. Analyse des mesures bathymétriques. $D=6.3 \mathrm{~cm}, \mathrm{KC}=7.9, \mathrm{G} / \mathrm{D}=1$, $\alpha=45^{\circ}$, a) Données brutes, b) Bathymétrie de grande échelle (morphodynamique),

c) Bathymétrie de petite échelie (rides et érosion).

Procedure of analysis of bed changes measurements. a). Raw data bed level,

b). Large scale morphodynamic variations, c).Ripples and scour bed changes

La soustraction de ces deux premiers champs permet d'évaluer les changements du lit sableux aux petites échelles, dus aux rides et à l'érosion (Figure 5c). Loin des piles, la hauteur des rides est de $20 \mathrm{~mm}$. En revanche, l'érosion autour des piles ne dépasse pas $5 \mathrm{~mm}$, valeur qui est en bon accord avec les résultats de Sumer et al. (1992) pour une pile isolée. Les figures 6-9 montrent les mêmes types de champs morphodynamiques que précédemment pour d'autres expériences avec $\mathrm{G} / \mathrm{D}=1$ mais pour différents nombres de $\mathrm{KC}$ et différentes orientations de vagues. 


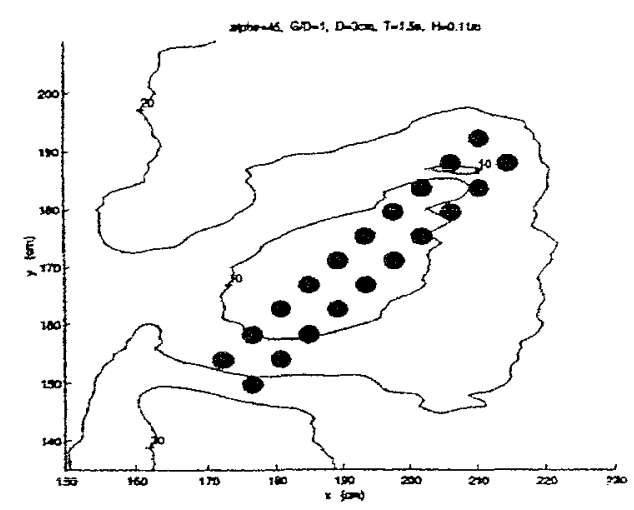

a)

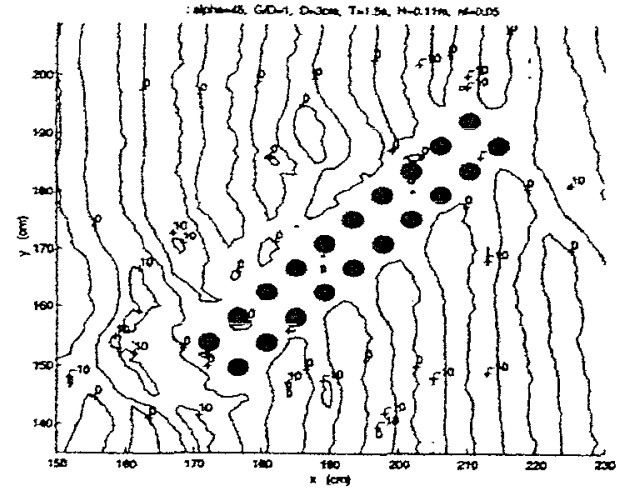

b)

Figure 6. $\mathrm{D}=3 \mathrm{~cm}, \mathrm{KC}=16.5, \mathrm{G} / \mathrm{D}=1, \alpha=45^{\circ}$, a) Bathymétrie de grande échelle (morphodynamique), b) Bathymétrie de petite échelle (rides et érosion) a) Large scale morphodynamic variations, b) Ripples and scour bed changes

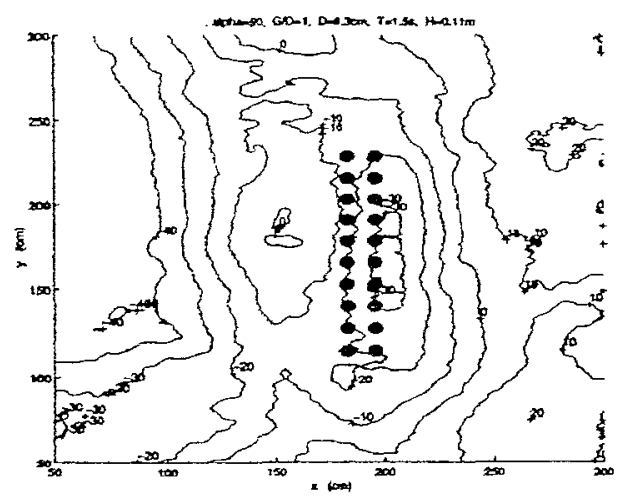

a)

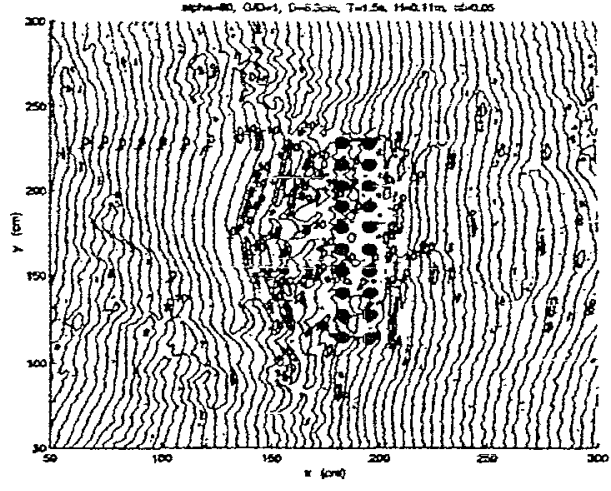

b)

Figure 7. $\mathrm{D}=6.3 \mathrm{~cm}, \mathrm{KC}=7.9, \mathrm{G} / \mathrm{D}=1, \alpha=90^{\circ}$, a) Bathymétrie de grande échelle (morphodynamique), b) Bathymétrie de petite échelle (rides et érosion).

a) Large scale morphodynamic variations, b) Ripples and scour bed changes 


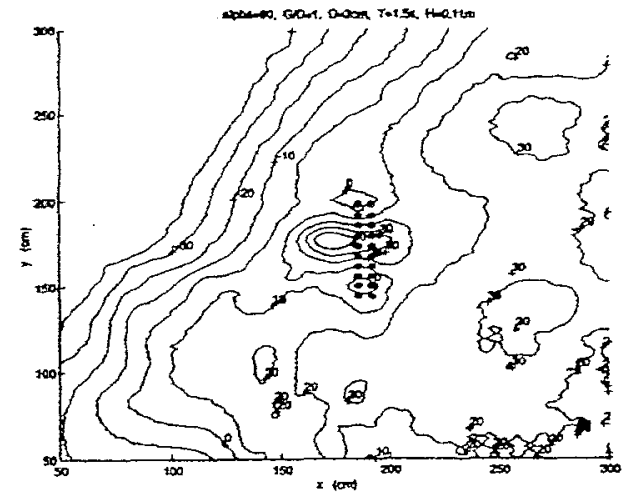

a)

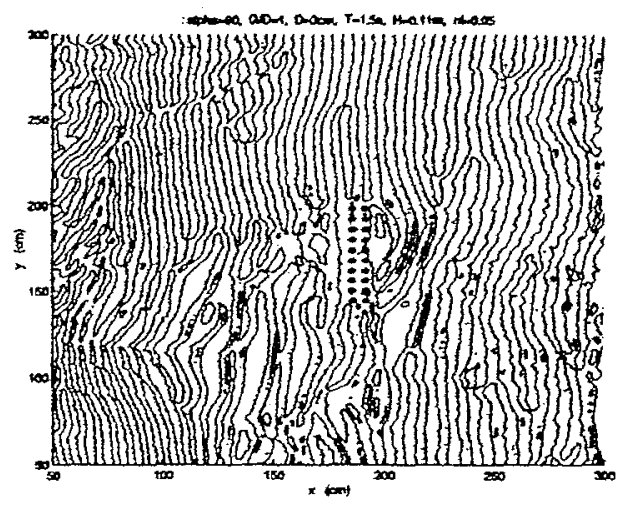

b)

Figure 8. $\mathrm{D}=3 \mathrm{~cm}, \mathrm{KC}=16.5, \mathrm{G} / \mathrm{D}=1, \alpha=90^{\circ}$, a) Bathymétrie de grande échelle (morphodynamique), b) Bathymétrie de petite échelle (rides et érosion).

a) Large scale morphodynamic variations, b) Ripples and scour bed changes

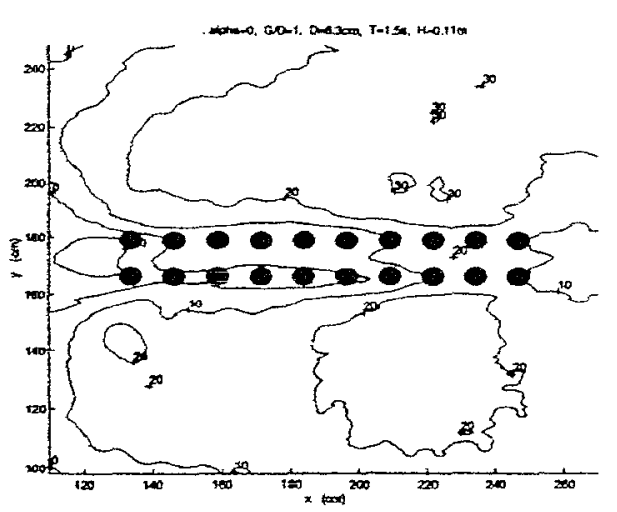

a)

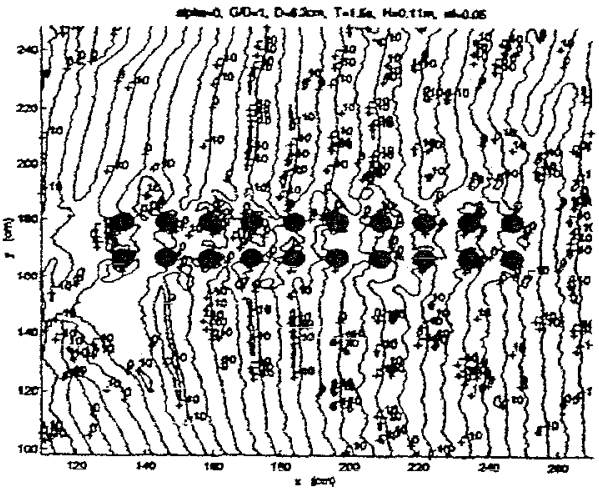

b)

Figure 9. $\mathrm{D}=6.3 \mathrm{~cm}, \mathrm{KC}=7.9, \mathrm{G} / \mathrm{D}=1, \alpha=0^{\circ}$, a) Bathymétrie de grande échelle (morphodynamique), b) Bathymétrie de petite échelle (rides et érosion).

a) Large scale morphodynamic variations, b) Ripples and scour bed changes

Les observations faites sur ces différentes expériences vont toutes dans le même sens et permettent de dégager les conclusions générales suivanies :

(i) Les modifications du lit sableux aux grandes échelles sont très marquées et varient d'un cas à l'autre en fonction de l'orientation et du nombre de Keulegan-Carpenter. Par exemple, pour l'incidence de $45^{\circ}$, on observe une profonde érosion globale pour $\mathrm{KC}=16.5$ (Fig. 6a) alors que pour un $\mathrm{KC}=7.9$ (Fig. 6b,7a) un dépôt est observé. De même, pour une incidence de $90^{\circ}$, une grande différence entre les bathymétries morphodynamiques de grande échelle est visible pour les deux KC (Fig. 7a,8a).

(ii) Des champs de rides réguliers sont observés dans toutes les expériences. $L$ 'amplitude des changements de fond des petites échelles est nettement plus faible au sein de piles que l'échelle des rides elle-même (Figures 5c, $6 b, 7 b, 8 b, 9 b)$. On note également que les rides sont atténuées au sein du groupe de piles. 
(i) L'érosion locale autour des piles est limitée dans nos expériences du fait de la faible gamme de $\mathrm{KC}$ possible (valeur maximale de $\mathrm{KC}$ de 16,5). Pour cette ordre de grandeur du nombre de Keleugan-Carpenter, Sumer et al. prévoient que la profondeur du trou autour d'une pile isolée est de 14.5 $\mathrm{mm}$. Dans nos expériences, la profondeur des trous d'érosion locale est de l'ordre de $10 \mathrm{~mm}$.

Nous présentons brièvement ci-dessous quelques observations de l'érosion autour d'une pile isolée placée initialement sur une plage de pente constante. L'originalité de ces expériences est que la pile était placée dans la zone de déferlement. En faisant varier la hauteur de la houle, différentes expériences ont été réalisées pour lesquelles la position du déferlement était modifiée pour se situer à différentes positions, à l'avant, au niveau de la pile, ou à l'arrière de la pile. La pile utilisée était une pile de grand diamètre $(\mathrm{D}=0,4 \mathrm{~m})$, afin d'avoir des valeurs du nombre de Keleugan-Carpenter faibles, significativement en dessous de la valeur limite $\mathrm{KC}=6$ pour laquelle les processus d'érosion locale apparaissent (Sumer et al. 1992). La figure 10 compare les variations bathymétriques morphodynamiques de grandes échelles pour deux situations de déferlement. Pour la Figure $10 \mathrm{~b}$ la ligne de déferlement est située $1 \mathrm{~m}$ à l'avant de la pile, tandis qu'elle est située à l'arrière de la pile pour le cas présenté sur la Figure 10a. Pour ces deux situations, la Figure 10 montre une forte érosion au voisinage de la pile associé à un processus global d'érosion, et non pas local. Lorsque le point de déferlement est situé à l'arrière de la pile (Figure 10a), un trou profond localisé à l'avant de la pile est observé, provoquant une déformation de la barre de déferlement. En revanche, lorsque le déferlement est situé à l'amont de la pile (Figure 10b), un trou d'érosion important est formé qui contient entièrement la pile. Les expériences sur l'érosion autour d'une pile située dans la zone de déferlement sont en cours d'analyse.

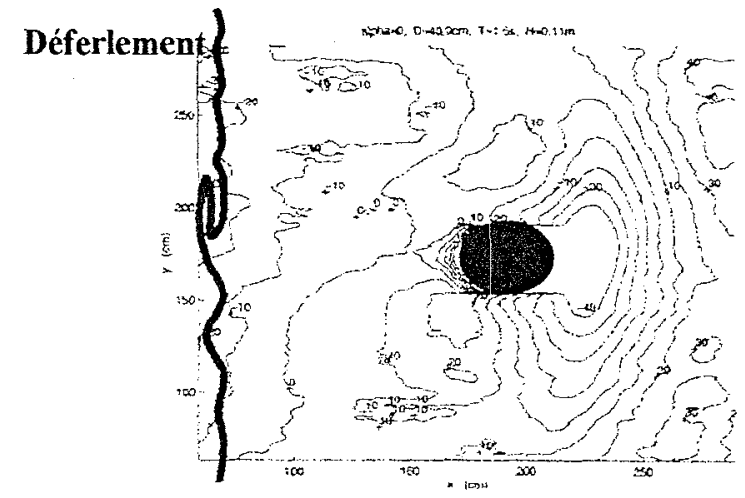

a)

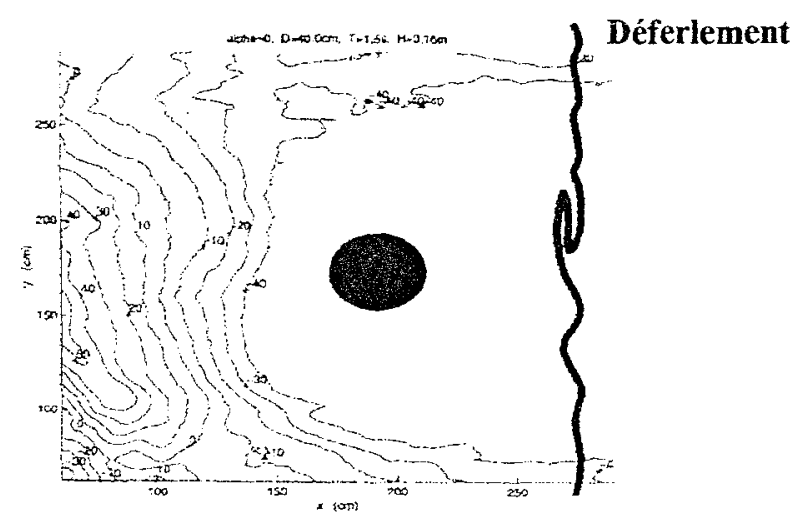

b)

Figure 10. $\mathrm{D}=40 \mathrm{~cm}, \mathrm{KC}=1.2$, Modifications bathymétriques de grandes échelles autour d'une pile située dans la zone de déferlement, a) $\mathrm{H}_{\mathrm{m}, \mathrm{d}}=0,11 \mathrm{~m}$,

b) $\mathrm{H}_{\mathrm{m}, \mathrm{d}}=0,16 \mathrm{~m}$

Morphodynamic large scale bed changes around a pile in the breaking zone, a) $H_{m, d}=0.11 \mathrm{~m}$, b) $H_{m, d}=0.16 \mathrm{~m}$ 


\section{Conclusion}

Une grande variété de formes de lits sableux a été observée dans des expériences en bassin à houle au voisinage d'un groupe de piles et au voisinage d'une pile isolée, lorsque cette dernière est située dans la zone de déferlement. Les groupes de piles étaient placés initialement sur une plage de pente constante et soumise à une houle régulière telle que le déferlement se produisait à l'arrière du groupe de piles. Les groupes de piles modifient de façon significative l'érosion à grandes échelles du fait de la diffraction de la houle. Les effets de plusieurs paramètres, en particulier l'incidence de la houle et le nombre de KeuleganCarpenter, sur l'érosion et les modifications de la bathymétrie à grandes et à petites échelles au voisinage des piles ont été étudiés. On a également fait varier le rapport entre la distance $\mathrm{G}$ entre deux piles et le diamètre $\mathrm{D}$ des piles

L'étude a permis de mettre en évidence que

- une érosion globale se superpose aux phénomènes d'érosion locale pour les configuration d'érosion au voisinage d'un groupe de piles,

- des rides de fond sont systématiquement observées. Les échelles respectives des profondeurs de rides et d'érosion ont été comparées.

- l'apparition d'une érosion profonde autour d'une pile isolée située dans la zone de déferlement est observée.

Nous mentionnons que l'utilisation des formules adimensionnelles reliant la profondeur d'érosion au nombre de Keleugan-Carpenter dans la perspective du dimensionnement d'ouvrages côtiers a été discutée ailleurs par Mory et al. (1999). De plus, au vue des premiers résultats dans la zone de déferlement, une étude plus approfondie des paramètres de ce problème va être faite pour mieux appréhender l'importance du courant de retour de fond sur l'affouillement de la pile de pont.

\section{Remerciements}

Cette étude a été réalisée dans le cadre du projet MAST-3 SCARCOST, financé en partie par la Commission Européenne, Direction Générale pour les Sciences, Contrat de Recherche-Développement MAS3-CT97-0097. La Société Sogréah Ingénierie et R. Gagnou sont remerciés pour leur assistance technique au Laboratoire d'Hydraulique de France, ainsi que Céline Laval pour son travail de stage, Benoit Camenen et Jean-Marc Barnoud du LEGI.

\section{Références}

LARRAS, J. 1972. Hydraulique et granulats. Collection du Centre de Recherche et d'Essais de Chatou, Eyrolles.

Herbisch, J.B. 1991. Scour around pipelines, piles and seawalls. in Handbook of Coastal and Ocean Engineering. 2: 867-958. Houston: Gulf Publishing Company. 
SUMER M., FREDSOE J. \& CHRISTIANSEN N. 1992. Scour around vertical pile in waves . ASCE J. Waterway, Port, Coastal and Ocean Engineering. 118(1): 15-31.

SUMER B.M. \& FREDSOE J. 1998. Wove scour around group of vertical piles. ASCE J. Waterway, Port, Coastal and Ocean Engineering. 124(5): 248-256.

SUTHERLAND J. \& WHTEHOUSE R.J.S. 1998. Scale effects in the Physical modelling of seabed scour. HR Wallingford Report TR64.

WHITEHOUSE R. J. S. 1998. Scour at marine structures, London: Thomas Telford.

Mory M., Larroude Ph., Carreiras J. \& Seabra Santos F.J., 1999 Scour around pile groups, Coastal Structures, Santander. 